\title{
COMPARACIÓN DE DOS PROTOCOLOS DE MANEJO EN PREECLAMPSIA SEVERA LEJOS DEL TÉRMINO, Y RESULTADOS MATERNOS Y NEONATALES: UNA COHORTE HISTÓRICA HOSPITAL UNIVERSITARIO SAN JOSÉ, POPAYÁN (COLOMBIA)
}

Comparison of two protocols for the management of early severe pre-eclampsia, and of materinal and neonatal outcomes: A historic cohort Hospitall Universitario San José, Popayán (Colombia)

César Augusto Rendón-Becerra, $M D^{1}$; Roberth Alirio Ortiz-Martínez, $M D^{2}$

Recibido: agosto 24/15 - Aceptado: marzo 14/16

\section{RESUMEN}

Objetivo: determinar si existe una diferencia en la seguridad del protocolo de manejo expectante o activo en gestantes con embarazos menores de 34 semanas y preeclampsia severa en resultados maternos y fetales.

Materiales y métodos: cohorte histórica de gestantes entre las 24 y 34 semanas, con diagnóstico de preeclampsia severa, que después de recibir un curso de corticoesteroides fueron manejadas con protocolo expectante o activo según criterio de equipo responsable en una institución universitaria de referencia en la región suroccidental de Colombia. Muestreo consecutivo. Se midió como

1 Residente de Ginecología y Obstetricia, Departamento de Medicina y Cirugía, Universidad del Cauca, Popayán (Colombia).

ceaure123@hotmail.com

2 Especialista en Ginecología y Obstetricia. Profesor, Departamento de Ginecología y Obstetricia, Mg. Epidemiología, Universidad del Cauca, Hospital Universitario San José, Popayán (Colombia). resultado primario la mortalidad y las complicaciones maternas, y una medida compuesta de pobres resultados maternos. Como resultados secundarios se midieron mortalidad neonatal, días de estancia hospitalaria en la unidad de cuidado intensivo neonatal (UCIN), horas de ventilación mecánica y una medida compuesta de complicaciones neonatales. Se compararon las características basales y clínicas de las dos poblaciones, y los resultados maternos y neonatales por medio de test exacto de Fisher o chi cuadrado para variables categóricas, y pruebas no paramétricas para variables continuas.

Resultados: los grupos fueron comparables con respecto a sus características basales. La prolongación del embarazo en el manejo expectante fue en promedio 8,8 días con DS +/- 8,74 días. No se presentaron diferencias estadísticas entre los dos protocolos respecto a la morbimortalidad materna o perinatal en la estancia hospitalaria posparto, los días de hospitalización del recién nacido y las horas de ventilación mecánica (p > 0,05). 
Conclusión: no se encontraron diferencias en términos de seguridad en resultados maternos y fetales con el manejo expectante en relación con el manejo activo en pacientes con preeclampsia severa temprana.

Palabras clave: preeclampsia, mortalidad perinatal, complicaciones del embarazo.

\section{ABSTRACT}

Objective: To determine if there is a difference in safety using expectant or active management for patients with less than 34 weeks of gestation and severe preeclampsia, in terms of maternal and foetal outcomes.

Materials and methods: Historic cohort of pregnant women between 24 and 34 weeks of gestation diagnosed with severe preeclampsia, managed in accordance with an expectant or an active protocol after a course of steroids, based on the judgment of the team in charge at a referral teaching hospital in the southwest of Colombia. Consecutive sampling. The primary endpoints measured were maternal mortality and complications, and a composite measurement of poor maternal outcomes. Secondary endpoints measured were neonatal mortality, length of stay in the neonatal intensive care unit (NICU) and hours of mechanical ventilation, as well as a composite measurement of neonatal complications. Baseline and clinical characteristics of the two populations were measured, as well as neonatal outcomes, using the exact Fisher test or the chi square test for categorical variables, and non-parametric tests for continuous variables.

Results: The groups were comparable in terms of their baseline characteristics. Pregnancy prolongation with expectant management was in average 8.8 days \pm 8.74 days SD. There were no statistical differences between the two protocols regarding maternal or perinatal morbidity and mortality during the postpartum period, neonatal length of stay, or mechanical ventilation hours $(p>0.05)$.
Conclusion: No advantages were found in terms of safety maternal and foetal outcomes when comparing expectant and active management of patients with early severe preeclampsia.

Key words: Pre-eclampsia, Perinatal mortality, Pregnancy complications.

\section{INTRODUCCIÓN}

La preeclampsia es una de las causas principales de morbi-mortalidad materna y perinatal y afecta al 6-12\% de los embarazos (1-3). Según la definición del American Congress of Obstetricians and Gynecologists (ACOG) la preeclampsia se define como tensión arterial $\geq 140 / 90 \mathrm{~mm} / \mathrm{Hg}$ que ocurre después de la semana 20 de gestación, con presiones arteriales previamente normales, con proteinuria $\geq 300 \mathrm{mg}$ en una recolección de orina de 24 horas $(4,5)$. Según la edad gestacional (EG) se clasifica en temprana y tardía; la temprana es aquella que se presenta antes de la semana 34 y la tardía después de esta (6).

La preeclampsia temprana se considera un trastorno asociado a disfunción placentaria, reducción en el volumen placentario, restricción del crecimiento intrauterino (RCIU), anormalidad en la evaluación del doppler de arteria umbilical, bajo peso al nacer, disfunción multiorgánica materna, resultados neonatales adversos, muerte intrauterina, un curso más severo de la enfermedad una mayor mortalidad materno-perinatal (7-9). A pesar de que los criterios diagnósticos de preeclampsia temprana y tardía son iguales, y los parámetros clínicos y biomarcadores son limitados, al parecer la preeclampsia temprana se relaciona más con factores placentarios intrínsecos, diferente de la tardía que se asocia más a los factores predisponentes maternos $(10,11)$. La insuficiencia en la placentación y la falla de la remodelación en las arterias espirales ocurre típicamente en la temprana, mientras que en la tardía se ha visto solo en una pequeña proporción de las placentas que muestran una remodelación incompleta en las arterias espirales (10-12). 
La preeclampsia severa se define, según la ACOG, por la presencia de uno de los siguientes signos: presión arterial sistólica $\geq 160 \mathrm{~mm} / \mathrm{Hg}$ o presión diastólica $\geq 110 \mathrm{~mm} / \mathrm{Hg}$, edema pulmonar, síntomas sugestivos de compromiso de órgano blanco (cefalea persistente, alteraciones visuales, dolor epigástrico o en hipocondrio derecho), oliguria $\leq 500 \mathrm{ml} / 24$ horas, hemolisis microangiopática, trombocitopenia, alteración de la función renal creatinina $\geq 1,1 \mathrm{mg} / \mathrm{dl}$, alteración de la función hepática (enzimas hepáticas dos veces por encima del valor normal) $(4,5)$.

En el manejo de la preeclampsia severa más allá de las 34 semanas de gestación existe el acuerdo de terminar el embarazo; sin embargo, en edades gestacionales menores la finalización de la gestación se asocia a un mayor riesgo de complicaciones neonatales por la prematurez (13), por lo que existe una controversia sobre los beneficios del manejo tradicional, según el cual, se requiere la terminación del embarazo una vez que se cumpla el ciclo de esteroides (13). Algunos autores proponen un manejo expectante en condiciones maternas no adversas una vez se complete el esquema de maduración pulmonar (esteroides), con el objetivo de mejorar los resultados neonatales y sin un mayor riesgo para la madre (14-19). Otros investigadores sugieren que este manejo podría tener mayor tasa de complicaciones y mayor frecuencia de resultados adversos maternos y fetales (20).

Teniendo en cuenta que la preeclampsia y sus complicaciones son un problema de salud pública debido a su impacto en la morbi-mortalidad tanto a corto como a largo plazo para la madre y para el neonato, además de la existencia de divergencias en el manejo de la preeclampsia severa menor de 34 semanas (manejo expectante frente a manejo activo), se propuso el presente estudio, con el objetivo de evaluar si hay diferencia en la seguridad de la aplicación del protocolo de manejo expectante o protocolo de manejo activo en mujeres con preeclampsia severa en términos de resultados maternos y perinatales.

\section{MATERIALES Y MÉTODOS}

Estudio de cohorte histórica, la cual estuvo constituida por pacientes con embarazo simple entre las 24 y 34 semanas, con diagnóstico de preeclampsia severa como criterios de inclusión, admitidas al Hospital Universitario San José Popayán (HUSJ), Cauca, centro de referencia de la región suroccidental de Colombia, entre 1 de enero del 2009 y 1 de enero del 2015. Se excluyeron las pacientes que al ingreso presentaban: eclampsia, síndrome HELLP (hemolisis caracterizada por LDH $\geq 600 \mathrm{U} / \mathrm{L}$, bilirrubina total $\geq 1,2 \mathrm{mg} / \mathrm{dl}$, elevación de enzimas hepáticas: aspartato aminotransferasa $\mathrm{AST} \geq 70 \mathrm{U} / \mathrm{L}$ y plaquetas $\leq 100.000$ células/mL), hipertensión severa no controlada ( $\geq 160 / 110 \mathrm{~mm} / \mathrm{Hg}$ ), abruptio de placenta, alteraciones fetales: desaceleraciones tardías repetitivas, desaceleraciones variables severas, variabilidad disminuida ( $\leq 3 \mathrm{~ms}$ ), oligoamnio severo (lago mayor $\leq 2 \mathrm{~cm}$, o índice de líquido amniótico $\leq 5 \mathrm{~cm}$ ), flujo diastólico reverso en la arteria umbilical en el doppler fetal.

Se realizó un muestro consecutivo revisando todas las historias clínicas en el periodo bajo estudio.

Procedimiento. Para la localización de la cohorte de pacientes se hizo una búsqueda en la unidad estadística del HUSJ con el código CIE 10 de ingreso de: trastorno hipertensivo del embarazo, preeclampsia, hipertensión arterial crónica e hipertensión materna. Se tomaron todas las gestantes con diagnóstico de preeclampsia severa de acuerdo con la definición antes mencionada del ACOG. La edad gestacional fue calculada mediante la fecha de la última menstruación confiable, ecografía del primer trimestre o ecografía realizada por el servicio de medicina materno-fetal.

A su ingreso, el manejo de estas pacientes incluía: accesos venosos, sonda vesical para el control estricto de líquidos administrados y eliminados, inicio temprano de esteroides, goteo de sulfato de magnesio con dosis de impregnación y mantenimiento durante las primeras 24 horas (según protocolo institucional); en presencia de crisis hipertensiva el tratamiento antihipertensivo se realizó con labetalol 
endovenoso como primera línea, en bolos intravenosos o en infusión continua; posteriormente, nifedipino o para mantener una tensión arterial entre 150/100 mm/Hg cuando fue necesario.

La vigilancia se realizó con monitorización continua no invasiva de signos vitales en las primeras 48 horas; al ingreso se tomó: hemograma completo, creatinina, enzimas hepáticas, tiempos de coagulación, bilirrubinas, deshidrogenasa láctica (LDH), proteinuria y depuración de creatinina en 24 horas. El recuento de plaquetas, hemograma, creatinina, enzimas hepáticas y LDH se hizo cada 24 a 72 horas dependiendo de los síntomas de la paciente. La condición fetal fue evaluada al ingreso con ecografía obstétrica, doppler de circulación feto-placentaria, perfil biofísico, nivel de líquido amniótico, cardiotocografía por turno de enfermería.

En el grupo de manejo expectante la vigilancia fetal se continuó cada 48-72 horas, excepto por el doppler que fue semanal por el servicio de medicina materno-fetal. El grupo de manejo activo, una vez se completó el ciclo de esteroides, fue llevado a terminación del embarazo; la vía de evacuación fue a criterio del grupo a cargo.

Las indicaciones fetales para la terminación del embarazo durante el manejo expectante fueron: alteraciones en la monitorización fetal, con desaceleraciones tardías repetitivas, desaceleraciones variables severas, variabilidad disminuida, oligoamnios severo, flujo diastólico reverso en la arteria umbilical, perfil biofísico $<4$ tomado en dos ocasiones con 4 horas de diferencia, RCIU con percentil de crecimiento menor a 5. Las indicaciones maternas fueron: hipertensión severa no controlada (definida como presión arterial $\geq 160 / 110 \mathrm{~mm}$ / $\mathrm{Hg}$, después de administrar dos antihipertensivos a dosis máximas), abruptio, persistencia de síntomas premonitorios (cefalea, visión borrosa, dolor epigástrico), trombocitopenia $<100.000$ células/ $\mathrm{mL}$, falla renal (definida como diuresis $<0,3 \mathrm{cc} / \mathrm{kg}$ / hora, creatinina $\geq 1,4 \mathrm{mg} / \mathrm{dl}$ ). La vía de evacuación fue a criterio del grupo a cargo.
El manejo posparto consistió en: sulfato de magnesio por 24 horas, terapia antihipertensiva según el caso, tromboprofilaxis, control estricto de líquidos, control paraclínico; el egreso se daba una vez la paciente se encontraba en situación estable.

Una vez seleccionadas las historias clínicas para incluir en este estudio, se extrajeron de ellas variables biológicas, sociales y demográficas mediante un cuestionario semiestructurado, que fue diseñado por los investigadores y revisado por expertos.

El resultado primario fue la mortalidad materna y una medida compuesta de pobres resultados maternos definidos como la presencia de, al menos, uno de los siguientes resultados: síndrome de Hellp, abruptio de placenta, ingreso a unidad de cuidado intensivo (UCI), edema pulmonar, falla renal, código rojo, necesidad de reintervención y muerte. En cuanto a los resultados fetales, se midieron: la muerte perinatal, las horas de ventilación mecánica y los días de estancia hospitalaria en Unidad de Cuidados Intensivos Neonatales (UCIN) y una medida compuesta de pobres resultados neonatales definidos como la presencia de, al menos, uno de los siguientes resultados: ingreso a UCIN, síndrome de dificultad respiratoria del recién nacido (SDRN), enterocolitis necrotizante, hemorragia intraventricular (HIV), peso al nacer, APGAR menor de 7 al primer y quinto minuto o muerte.

Para el análisis estadístico se creó una base de datos con control de ingreso a través de reglas de validación, a fin de garantizar la confiabilidad de estos (programación Excel); posteriormente, se llevó a Stata 10.0. La información se analizó desde el punto de vista exploratorio para mirar la normalidad de su distribución, identificar valores extremos y valores perdidos que pudieran incidir en el resultado; la población se describió de acuerdo con características sociales, demográficas y biológicas. La comparación de los dos grupos respecto a los datos dicotómicos se hizo por medio de la prueba chi cuadrado o test exacto de Fisher, las variables continuas que presentaron una distribución normal se analizaron mediante 
una t de Student, y para aquellas con distribución no normal se utilizó U de Mann-Whitney, el valor de p que se tomó como significante fue $<0,05$.

El presente estudio fue aprobado por el comité de ética médica de la institución.

\section{RESULTADOS}

Entre el 1 de enero de 2009 al 1 de enero del 2015 se identificaron 756 pacientes con diagnóstico de preeclampsia severa, de las cuales $656(86,1 \%)$ tenían una edad gestacional mayor a 34 semanas. Cien pacientes cumplieron con los criterios de inclusión; de estas, 34 fueron excluidas debido a que por la condición materna o fetal debieron ser evacuadas antes del ingreso a protocolo (abruptio de placenta, eclampsia, bienestar fetal no satisfactorio, entre otros). Un total de 66 pacientes cumplieron con los criterios de inclusión; después de cumplir el curso de esteroides, 35 pacientes $(53,1 \%)$ fueron manejadas con protocolo activo y 31 pacientes $(46,9 \%)$ con protocolo expectante; el análisis final se realizó con este grupo de pacientes (figura 1).

En el grupo de manejo activo la edad promedio fue de 26,6 años con DS de 7,9 años, y para el expectante fue de 27,2 con una DS de 8,0 años. La edad gestacional al ingreso para el grupo de manejo activo fue de 230,42 días con una DS de 12,16 días y para el expectante fue 223,87 días con una DS de 14,73 días; al comparar los grupos con respecto a estas variables no se encontraron diferencias estadísticas $(p>0,05)$. En lo referente a los aspectos sociodemográficos se encontró diferencia estadística en cuanto a la procedencia. Con respecto a las otras variables basales analizadas, tanto sociodemográficas como paraclínicas, no se encontraron diferencias estadísticamente significativas $(\mathrm{p}>0,05)$ (tabla 1).

Figura 1.

Selección de pacientes con diagnóstico de preeclampsia severa

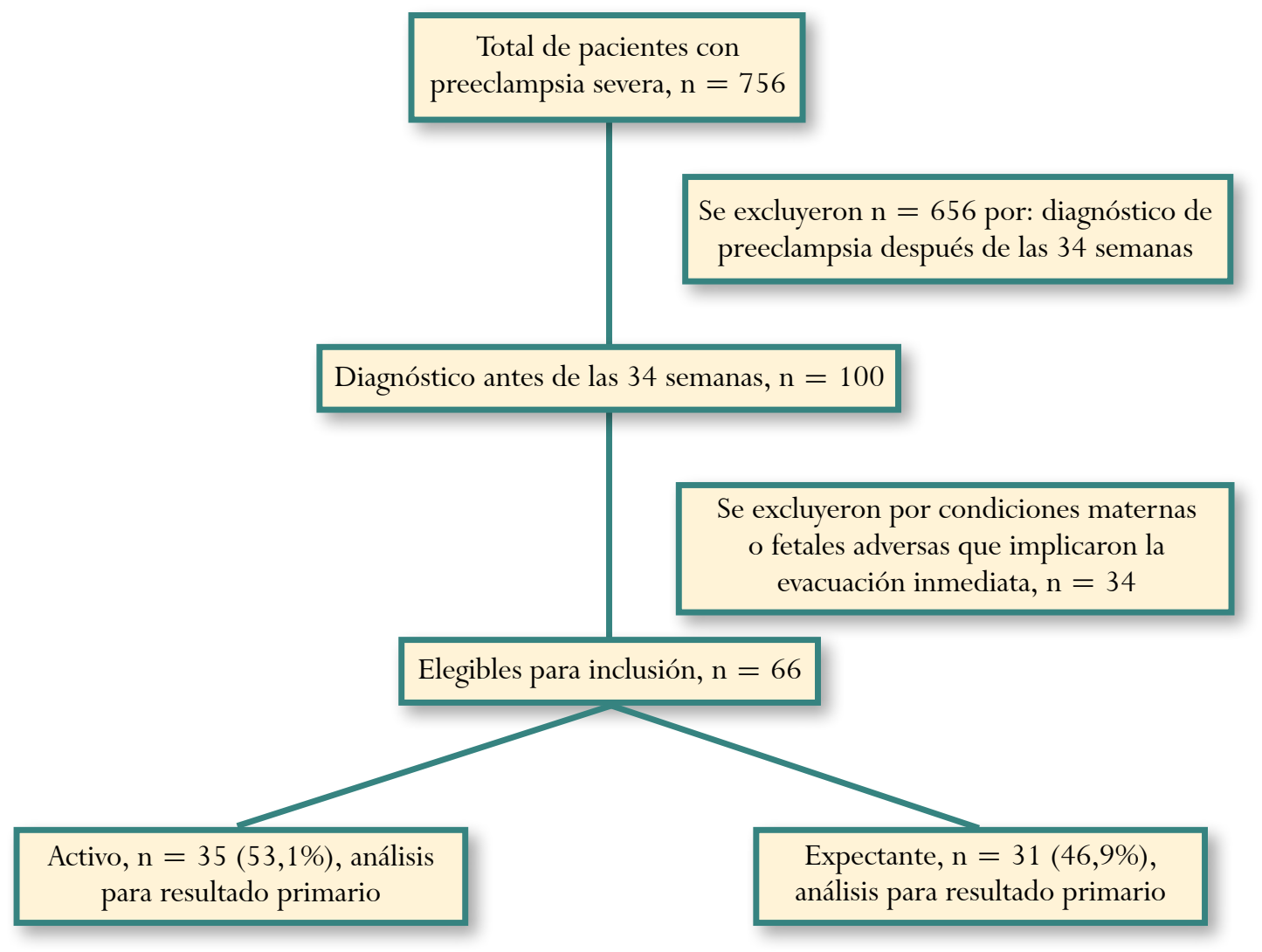

Fuente: estudio. 
Respecto a los malos desenlaces maternos se presentó una muerte materna, una eclampsia, dos casos de síndrome Hellp y un abruptio de placenta en el manejo expectante. En el grupo de manejo activo se presentaron 4 casos de síndrome Hellp y un caso de falla renal; sin embargo, estas diferencias no fueron significativas entre los dos protocolos como tampoco lo fue el resultado primario compuesto materno (tabla 2). La prolongación del embarazo en el manejo expectante fue en promedio 8,8 días con DS +/- 8,74 días.

Respecto a los resultados perinatales hubo una muerte neonatal en el manejo expectante, y aunque la estancia hospitalaria y las horas de ventilación del recién nacido fueron mayores en el grupo expectante, estas diferencias no fueron estadísticamente significativas. El resultado complejo neonatal no mostró diferencias entre los dos grupos ( $p>0,05)$ (tabla 3).

\section{DISCUSIÓN}

Los hallazgos de este estudio indican que no existe diferencia respecto a los resultados maternos y neonatales, al comparar el manejo expectante con el activo. Estos resultados son consistentes con los informados por Vigil de Gracia en un ensayo clínico con 267 pacientes en ocho instituciones latinoamericanas de tercer nivel, en donde concluyen que el manejo expectante no demuestra beneficios neonatales y, adicionalmente, aumenta el riesgo de abruptio de placenta y de que sea pequeño para la edad gestacional (PEG) (20). A diferencia de nuestros resultados, Sibai et al., en un ensayo clínico aleatorizado, concluyen que el manejo expectante en una institución de tercer nivel está asociado con buen resultado perinatal, menor estancia neonatal y con mínimo riesgo para la madre (16); de la misma manera, Odendaal et al., resaltan que el número de neonatos que requieren ventilación mecánica es menor en el expectante, con una menor frecuencia de complicaciones neonatales (18). Por otra parte, revisiones sistemáticas sugieren que un enfoque expectante podría asociarse con una disminución de la morbilidad neonatal; sin embargo, esta evidencia se basa en datos de solo cuatro ensayos clínicos y se necesitan ensayos clínicos aleatorizados más grandes para confirmar o refutar estos resultados y establecer si este enfoque es seguro para la madre (21).

El presente estudio tiene como fortalezas: criterios de inclusión y exclusión claros, resultados maternos y fetales adecuadamente definidos y existencia de protocolos institucionales estandarizados y no pérdidas en el seguimiento, lo cual permite reducir el riesgo de desempeño y detección entre los dos grupos por parte de los médicos tratantes y el sesgo derivado de datos ausentes. Como debilidades, ser una cohorte histórica en donde la decisión de aplicar un protocolo estuvo a cargo de los médicos tratantes, lo cual puede generar sesgos de selección. Con respecto al tamaño de muestra, es de anotar que es pequeño y es posible que no se encontraran diferencias, no porque no las haya, sino porque el estudio no tiene el poder suficiente para encontrarlas (potencia menor a un $80 \%$ ). Sin embargo, hay varias investigaciones con tamaños de muestra similares (16-20). Por último, no se hizo ajuste por posibles factores de confusión conocidos tales como la edad materna o gestacional o la vía del parto.

\section{CONCLUSIÓN}

No se encontraron diferencias en resultados maternos y neonatales al comparar los dos protocolos, no habiendo ventajas con el manejo expectante. Se sugiere la realización ensayos clínicos aleatorizados con adecuado tamaño de muestra, que den evidencia de mejor calidad para definir la seguridad del manejo expectante y del activo.

\section{REFERENCIAS}

1. Conde-Agudelo A, Belizán JM, Lammers C. Maternalperinatal morbidity and mortality associated with adolescent pregnancy in Latin America: Cross-sectional study. Am J Obstet Gynecol. 2005;192:342-9.

2. Cousens S, Blencowe H, Stanton C, Chou D, Ahmed S, Steinhardt L, et al. National, regional, and worldwide estimates of stillbirth rates in 2009 with trends since 1995: a systematic analysis. Lancet. 2011;377:1319-30. 
Tabla 1.

Características basales de población en estudio, comparación de dos protocolos de manejo de preeclampsia severa en gestantes con embarazo menor de 34 semanas, HUSJ, Popayán, 2009-2015

\begin{tabular}{|c|c|c|c|}
\hline Variable & $\begin{array}{c}\text { Protocolo activo } \\
\mathbf{n}=35 \\
\mathbf{n}(\%)\end{array}$ & $\begin{array}{l}\text { Protocolo expectante } \\
\qquad \begin{array}{c}\mathbf{n}=31 \\
\mathbf{n}(\%)\end{array}\end{array}$ & Valor $\mathbf{p}$ \\
\hline \multicolumn{4}{|l|}{ Edad (años) } \\
\hline$\leq 19$ & $8(22,86)$ & $7(22,58)$ & 0,91 \\
\hline $20-34$ & $19(54,29)$ & $17(54,84)$ & \\
\hline$\geq 35$ & $8(22,86)$ & $7(22,58)$ & \\
\hline \multicolumn{4}{|l|}{ Residencia } \\
\hline Urbana & $14(40)$ & $20(64,52)$ & 0,04 \\
\hline Rural & $21(60)$ & $11(35,48)$ & \\
\hline \multicolumn{4}{|l|}{ Seguridad social } \\
\hline Contributivo & $6(17,14)$ & $7(22,58)$ & 0,57 \\
\hline Subsidiado & $29(82,86)$ & $24(77,42)$ & \\
\hline \multicolumn{4}{|l|}{ Control prenatal $^{1}$} \\
\hline Adecuado & $18(51,43)$ & $17(54,84)$ & 0,78 \\
\hline No adecuado & $17(48,57)$ & $14(45,16)$ & \\
\hline Edad gestacional en días al ingreso* & $230,42 \pm 12,16$ & $223,87 \pm 14,73$ & 0,06 \\
\hline Plaquetas $(\mathrm{n} / \mathrm{mm} 3) *$ & $198571 \pm 56514$ & $221032 \pm 48840$ & 0,09 \\
\hline LDH $(\mathrm{U} / \mathrm{L})^{*}$ & $231,77 \pm 5,24$ & $230,61 \pm 4,16$ & 0,21 \\
\hline $\operatorname{AST}(\mathrm{U} / \mathrm{L}) *$ & $33,14 \pm 9,54$ & $31,22 \pm 9,15$ & 0,25 \\
\hline $\operatorname{ALT}(\mathrm{U} / \mathrm{L}) *$ & $31,37 \pm 3,03$ & $30,93 \pm 4,35$ & 0,74 \\
\hline Creatinina $(\mathrm{mg} / \mathrm{dl})^{*}$ & $0,8 \pm 0,21$ & $0,70 \pm 0,15$ & 0,81 \\
\hline TP segundos* & $7,95 \pm 0,78$ & $8,14 \pm 0,94$ & 0,41 \\
\hline TPT segundos* & $29,77 \pm 2,42$ & $28,09 \pm-3,65$ & 0,06 \\
\hline Bilirrubinas total $(\mathrm{mg} / \mathrm{dl})^{*}$ & $0,51 \pm 0,14$ & $0,45 \pm 0,14$ & 0,07 \\
\hline Proteínas (mg)* & $1117 \pm 443$ & $1680 \pm 907$ & 0,24 \\
\hline
\end{tabular}

Fuente: estudio.

$1=$ CPN: control prenatal no adecuado definido por la OMS con un valor $\leq 4$ controles

* media \pm desviación estándar (DS) 
Tabla 2.

Resultados maternos, comparación de dos protocolos de manejo de preeclampsia severa en gestantes con embarazo menor de 34 semanas, HUSJ, Popayán, 2009-2015

\begin{tabular}{|c|c|c|c|}
\hline Variable & $\begin{array}{l}\text { Protocolo activo } \\
\qquad \begin{array}{c}\mathbf{n}=35 \\
\mathbf{n}(\%)\end{array}\end{array}$ & $\begin{array}{c}\text { Protocolo expectante } \\
\qquad \mathbf{n}=31 \\
\mathbf{n}(\%)\end{array}$ & Valor $\mathbf{p}$ \\
\hline $\begin{array}{c}\text { Días de estancia posparto } \\
\text { mediana (rango intercuartílico) }\end{array}$ & $3(3-4)$ & $3(2-4)$ & 0,176 ा \\
\hline $\begin{array}{c}\text { Abruptio placenta } \\
\text { No } \\
\text { Sí }\end{array}$ & $\begin{array}{c}35(100) \\
0(0)\end{array}$ & $\begin{array}{c}30(96,77) \\
1(3,23)\end{array}$ & $0,47^{*}$ \\
\hline $\begin{array}{l}\text { Sd. Hellp } \\
\text { No } \\
\text { Sí }\end{array}$ & $\begin{array}{l}31(88,57) \\
4(11,43)\end{array}$ & $\begin{array}{c}29(93,55) \\
2(6,45)\end{array}$ & $0,67^{*}$ \\
\hline $\begin{array}{c}\text { Edema pulmonar } \\
\text { No } \\
\text { Sí }\end{array}$ & $\begin{array}{c}35(100) \\
0(0)\end{array}$ & $\begin{array}{c}31(100) \\
0(0)\end{array}$ & $\mathrm{n} / \mathrm{a}$ \\
\hline $\begin{array}{l}\text { Falla renal } \\
\text { No } \\
\text { Sí }\end{array}$ & $\begin{array}{c}34(97,14) \\
1(2,86)\end{array}$ & $\begin{array}{l}31(100) \\
0(100)\end{array}$ & $1,00^{*}$ \\
\hline $\begin{array}{l}\text { Código Rojo } \\
\text { No } \\
\text { Sí }\end{array}$ & $\begin{array}{c}34(97,14) \\
1(2,86)\end{array}$ & $\begin{array}{c}30(96,77) \\
1(3,23)\end{array}$ & $0,72^{*}$ \\
\hline $\begin{array}{c}\text { Reintervención quirúrgica } \\
\text { No } \\
\text { Sí }\end{array}$ & $\begin{array}{c}35(100) \\
0(0)\end{array}$ & $\begin{array}{c}29(93,55) \\
2(6,45)\end{array}$ & $0,21^{*}$ \\
\hline $\begin{array}{l}\text { Eclampsia } \\
\text { No } \\
\text { Si }\end{array}$ & $\begin{array}{c}35(100) \\
0(0)\end{array}$ & $\begin{array}{c}30(96,77) \\
1(3,23)\end{array}$ & $0,47^{*}$ \\
\hline $\begin{array}{l}\text { Muerte materna } \\
\text { No } \\
\text { Sí }\end{array}$ & $\begin{array}{c}35(100) \\
0(0)\end{array}$ & $\begin{array}{c}30(96,77) \\
1(3,23)\end{array}$ & $0,47^{*}$ \\
\hline $\begin{array}{l}\text { Ingreso a UCI } \\
\text { No } \\
\text { Sí }\end{array}$ & $\begin{array}{l}31(88,57) \\
4(11,43)\end{array}$ & $\begin{array}{c}26(83,87) \\
5(16,13)\end{array}$ & $0,72^{*}$ \\
\hline $\begin{array}{c}\text { Resultado compuesto materno } † \dagger \\
\text { No } \\
\text { Sí }\end{array}$ & $\begin{array}{l}27(77,14) \\
8(22,86)\end{array}$ & $\begin{array}{c}25(80,65) \\
6(19,35)\end{array}$ & $0,77^{* * *}$ \\
\hline
\end{tabular}

Fuente: estudio n/a: no aplica, * Test de Fisher, ** Chi cuadrado, $\uparrow$ U de Mann Whitney, †† Una misma paciente puede tener más de una complicación, se tomó solo el evento principal. 
Tabla 3.

Resultados neonatales, comparación de dos protocolos de manejo de preeclampsia severa en gestantes con embarazo menor de 34 semanas, HUSJ, Popayán, 2009-2015

\begin{tabular}{|c|c|c|c|}
\hline Variable & $\begin{array}{l}\text { Protocolo activo } \\
\qquad \mathbf{n}=35 \\
\mathbf{n}(\%)\end{array}$ & $\begin{array}{c}\text { Protocolo expectante } \\
\mathbf{n}=31 \\
\mathbf{n}(\%)\end{array}$ & Valor $\mathbf{p}$ \\
\hline $\begin{array}{l}\text { Días de hospitalización UCIN } \\
\text { mediana (rango intercuartílico) }\end{array}$ & $6(0-14)$ & $9(0-15)$ & 0,39 ฯ \\
\hline $\begin{array}{l}\text { Horas de ventilación mecánica } \\
\text { mediana (rango intercuartílico) }\end{array}$ & $72(48-96)$ & $108(30-408)$ & 0,34 ๆ \\
\hline $\begin{array}{l}\text { Muerte neonatal } \\
\text { No } \\
\text { Sí }\end{array}$ & $\begin{array}{c}35(100) \\
0(0)\end{array}$ & $\begin{array}{c}30(96,77) \\
1(3,23)\end{array}$ & $0,47^{*}$ \\
\hline $\begin{array}{l}\text { Enterocolitis } \\
\text { No } \\
\text { Sí }\end{array}$ & $\begin{aligned} 32 & (91,43) \\
3 & (8,57)\end{aligned}$ & $\begin{array}{c}30(96,77) \\
1(3,23)\end{array}$ & $0,60^{*}$ \\
\hline $\begin{array}{l}\text { Sind. dificultad respiratoria } \\
\text { No } \\
\text { Sí }\end{array}$ & $\begin{array}{l}13(37,14) \\
22(62,86)\end{array}$ & $\begin{array}{l}15(48,39) \\
16(51,61)\end{array}$ & $0,35^{* *}$ \\
\hline $\begin{array}{c}\text { Hemorragia intraventricular } \\
\text { No } \\
\text { Sí }\end{array}$ & $\begin{array}{c}34(97,14) \\
1(2,86)\end{array}$ & $\begin{array}{c}30(96,77) \\
1(3,23)\end{array}$ & $0,72^{*}$ \\
\hline $\begin{array}{l}\text { Enf. membrana hialina } \\
\text { No } \\
\text { Sí }\end{array}$ & $\begin{array}{l}28(80,0) \\
7(20,00)\end{array}$ & $\begin{array}{l}23(74,19) \\
8(25,81)\end{array}$ & $0,57^{* *}$ \\
\hline $\begin{array}{c}\text { Bajo peso para el EG } \\
\text { No } \\
\text { Sí }\end{array}$ & $\begin{array}{l}13(37,14) \\
22(62,86)\end{array}$ & $\begin{array}{l}10(32,26) \\
21(67,74)\end{array}$ & $0,67^{* *}$ \\
\hline $\begin{array}{c}\text { Otras complicaciones asociadas } \\
\text { No } \\
\text { Sí }\end{array}$ & $\begin{array}{c}26(74,29) \\
9(25,71)\end{array}$ & $\begin{array}{l}18(58,06) \\
13(41,94)\end{array}$ & $0,16^{* *}$ \\
\hline $\begin{array}{c}\text { apgar } \leq 7 \text { al } 1 \mathrm{~min} \\
\text { No } \\
\text { Sí }\end{array}$ & $\begin{array}{l}24(68,57) \\
11(31,43)\end{array}$ & $\begin{array}{c}26(83,87) \\
5(16,13)\end{array}$ & $0,14^{* *}$ \\
\hline $\begin{array}{c}\text { apgar } \leq 7 \text { a } 5 \mathrm{~min} \\
\text { No } \\
\text { Sí }\end{array}$ & $\begin{array}{l}27(77,14) \\
8(22,86)\end{array}$ & $\begin{array}{c}29(93,55) \\
2(6,45)\end{array}$ & $0,06^{*}$ \\
\hline $\begin{array}{c}\text { Ventilación mecánica } \\
\text { No } \\
\text { Sí }\end{array}$ & $\begin{array}{l}22(62,86) \\
13(37,14)\end{array}$ & $\begin{array}{l}21(67,74) \\
10(32,26)\end{array}$ & $0,67^{* *}$ \\
\hline $\begin{array}{c}\text { Resultado compuesto } \ddagger \\
\text { No } \\
\text { Sí }\end{array}$ & $\begin{array}{l}11(31,43) \\
24(68,57)\end{array}$ & $\begin{array}{l}12(38,71) \\
19(61,29)\end{array}$ & $0,53^{* *}$ \\
\hline
\end{tabular}

Fuente: estudio. *Test de Fisher, ** Chi cuadrado, $\uparrow$ U de Mann Whitney. $\ddagger$ Un neonato puede tener más de una complicación, se tomó solo el evento principal. 
3. Khan KS, Wojdyla D, Say L, Gülmezoglu AM, Van Look PFA. WHO analysis of causes of maternal death: a systematic review. Lancet. 2006;367:1066-74.

4. ACOG Committee on Practice Bulletins-Obstetrics. ACOG practice bulletin. Diagnosis and management of preeclampsia and eclampsia. Number 33, January 2002. Obstet Gynecol. 2002;99:159-67.

5. Hypertension in Pregnancy: Executive Summary. Obstet Gynecol. 2013;122:1122-31.

6. Raymond D, Peterson E. A critical review of earlyonset and late-onset preeclampsia. Obstet Gynecol Surv. 2011;66:497-506.

7. Hladunewich M, Karumanchi SA, Lafayette R. Pathophysiology of the clinical manifestations of preeclampsia. Clin J Am Soc Nephrol CJASN. 2007;2:543-9.

8. Kanasaki K, Kalluri R. The biology of preeclampsia. Kidney Int. 2009;76:831-7.

9. Askie LM, Duley L, Henderson-Smart DJ, Stewart LA, PARIS Collaborative Group. Antiplatelet agents for prevention of pre-eclampsia: a meta-analysis of individual patient data. Lancet. 2007;369:1791-8.

10. Li XL, Guo PL, Xue Y, Gou WL, Tong M, Chen Q. An analysis of the differences between early and late preeclampsia with severe hypertension. Pregnancy Hypertens Int J Womens Cardiovasc Health. 2016; $6: 47-52$.

11. Stergiotou I, Crispi F, Valenzuela-Alcaraz B, Bijnens B, Gratacos E. Patterns of maternal vascular remodeling and responsiveness in early- versus late-onset preeclampsia. Am J Obstet Gynecol. 2013;209:558.e1558.e14.

12. Ogge G, Chaiworapongsa T, Romero R, Hussein Y, Kusanovic JP, Yeo L, et al. Placental lesions associated with maternal underperfusion are more frequent in early-onset than in late-onset preeclampsia, J Perinat Med. 2011;39:641-52.
13. Sibai BM, Spinnato JA, Watson DL, Hill GA, Anderson GD. Pregnancy outcome in 303 cases with severe preeclampsia. Obstet Gynecol. 1984;64:319-25.

14. Haddad B, Sibai BM. Expectant management in pregnancies with severe pre-eclampsia. Semin Perinatol. 2009;33:143-51.

15. Publications Committee, Society for Maternal-Fetal Medicine, Sibai BM. Evaluation and management of severe preeclampsia before 34 weeks' gestation. Am J Obstet Gynecol. 2011;205:191-8.

16. Sibai BM, Mercer BM, Schiff E, Friedman SA. Aggressive versus expectant management of severe preeclampsia at 28 to 32 weeks gestation: a randomized controlled trial. Am J Obstet Gynecol 1994;171:818-22.

17. Haddad B, Deis S, Sibai B. Maternal and perinatal outcomes during expectant management of 239 severe preeclamptic women between 24 and 33 weeks' gestation. Am J Obstet Gynecol. 2004;190:1590 e7.

18. Odendaal HJ, Pattinson RC, Bam R, Grove D, Kotze TJ. Aggressive or expectant management for patients with severe preeclampsia between 28-34 weeks' gestation: a randomized controlled trial. Obstet Gynecol. 1990;76:1070-5.

19. Sarsam DS, Shamden M, Al Wazan R. Expectant versus aggressive management in severe preeclampsia remote from term. Singapore Med J. 2008;49:698-703.

20. Vigil-De Gracia P, Tejada OR, Minaca AC, et al. Expectant management of severe preeclampsia remote from term: a randomized, multicenter clinical trial: the Mexpre Latin Study. Am J Obstet Gynecol. 2013;209:425.e1-8.

21. Churchill D, Duley L, Thornton JG, Jones L. Interventionist versus expectant care for severe preeclampsia between 24 and 34 weeks' gestation (Review). The Cochrane Collaboration; 2013. 